\title{
Near vision anomalies in Black high school children in Empangeni, South Africa: A pilot study
}

\author{
Sam O Wajuihian* and Rekha Hansraj ${ }^{\dagger}$
}

<swajuihian@mweb.co.za >

${ }^{*}{ }^{\dagger}$ Discipline of Optometry, School of Health Sciences, University of KwaZulu-Natal, Private Bag X54001, Durban, 4000 South Africa

Received 8 October 2013; revised version accepted 18 March 20144

\section{Abstract}

Background: The ability to read efficiently and comfortably is important in the intellectual development and academic performance of a child. Some children experience difficulties when reading due to symptoms related to near vision anomalies.

Aim: To explore the feasibility of conducting a large study to determine the prevalence, distribution and characteristics of near vision anomalies in high school children in Empangeni, South Africa.

Methods: The study was a cross sectional descriptive pilot study designed to provide preliminary data on prevalence, distribution and characteristics of near vision anomalies in a sample of high school-children in South Africa. Study participants comprised 65 Black children (30 males and 35 females), ages ranged between 13 and 19 years with a mean age and standard deviation of $17 \pm 1.43$ years. The visual functions evaluated and the techniques used included visual acuity (LogMAR acuity chart), refractive error (autorefractor and subjective refraction), heterophoria (von Graefe), near point of convergence (push-in-to-double), amplitude of accommodation (push-in-to-blur) accommodation facility ( $\pm 2 \mathrm{D}$ flipper lenses), relative accommodation, accommodation response (monocular estimation method) and fusional vergences (step vergence with prism bars). Possible associations between symptoms and near vision anomalies were explored using a 20-point symptoms questionnaire.

Results: Prevalence estimates were: Myopia $4.8 \%$, hyperopia $1.6 \%$ and astigmatism $1.6 \%$. For accommodative anomalies, $1.6 \%$ had accommodative insufficiency while $1.6 \%$ had accommodative infacility. For convergence anomalies, $3.2 \%$ had receded near point of convergence, $16 \%$ had low suspect convergence insufficiency, no participant had high suspect convergence insufficiency, $1.6 \%$ had definite convergence insufficiency and $3.2 \%$ had convergence excess. Female participants reported more symptoms than the males and the association between clinical measures and symptoms were not remarkable.

Conclusion: Although the overall prevalence of near vision anomalies was low, convergence anomalies were found to be more prevalent than refractive and accommodative anomalies. Symptoms were not significantly associated with near vision anomalies. Overall, the pilot project reveals that it is feasible to conduct the study on a large scale with minor modifications. Identification and referrals for near vision anomalies are important steps towards diagnosis and treatment which will minimize discomfort with reading and subsequent poor performance which may be associated with such anomalies. ( $S$ Afr Optom 2014 73(1) 21-32)

Key words: Refractive errors, convergence insufficiency, convergence excess, accommodative insufficiency, accommodative excess, near vision anomalies in school children 


\section{Introduction}

The ability to read efficiently and comfortably is important in the intellectual development and academic performance of a child ${ }^{1}$. Often, optometrists see patients with complaints of blurred vision at near, diplopia, headaches, eye strain, watery eyes and ocular fatigue during reading. These symptoms, which are often related to near vision anomalies (NVAs) create discomfort and impair efficient near tasks including reading, writing or computer-based work $^{1-2}$. For the school-aged child, especially those in high school, such symptoms tend to increase as the child progresses through school when there is greater demand on the accommodative and vergence system for sustained clear vision due to prolonged reading and increased information processing ${ }^{1,2}$. Near vision anomalies affect clarity, binocularity, impair comfort and efficiency of visual performance of an individual particularly when near tasks are performed ${ }^{1,3}$. Aspects of near vision anomalies of interest in the present study include refractive errors (myopia, hyperopia, astigmatism and anisometropia), accommodative (accommodative insufficiency, excess and infacility) and vergence (convergence insufficiency and/ or excess, fusional vergence dysfunction and heterophoria). Reading is the process of extracting meaning from written text and it is a fundamental aspect of learning and hence education of a child 5. An adequate interaction and coordination of the refractive, accommodative and vergence systems is essential to read efficiently ${ }^{1}$. Rity et $a l^{6}$ found that of the total amount of time a child spends at school on academic activities, 54\% was spent doing near work, tasks that require shifting focus from distance to near and from near to distance took $21 \%$ of the time, while $25 \%$ was spent on distance work. A traditional approach to identifying vision anomalies in the school setting is through vision screenings. Invariably, most school vision screenings are inadequate as most eye tests performed focus on visual acuity and refractive errors $^{7}$ giving less emphasis to visual parameters related to NVAs. Near vision anomalies have various negative consequences ${ }^{8-17}$. Convergence insufficiency (CI) has been associated with attention deficit hyperactivity disorder (ADHD) ${ }^{8-11}$, anxiety, emotional and social problems ${ }^{13-14}$ and lowered academic achievements ${ }^{14-15}$. In addition, NVAs are prevalent in populations of children with reading difficulties ${ }^{12,16}$ and dyslexia ${ }^{17}$.

Although the assessment of an individual's binocular vision status related to NVAs constitutes an important part of optometric practice, studies in this area of eye care show diverse findings due to the methodological challenges in conducting such studies in a school setting on a large scale ${ }^{18-19}$. However, studies ${ }^{20-44}$ have attempted to characterize various aspects of NVAs in unselected populations of school children though no available studies that related refractive errors to near vision functions could be found. Accommodative anomalies have been reported but mainly on accommodative insufficiencies (AI). The prevalence of $\mathrm{AI}$ using various criteria ranged between $2.3 \%$ and $24 \% 26,28,30,32-35$, accommodative excess ranged between $1.8-3.7 \% 12,32$ while accommodative infacility (AIF) and poor accommodative facility ranged between $12.3 \%$ and $53 \%{ }^{32-35}$. For convergence anomalies, CI was most studied ${ }^{24,30,37-40,43}$ and was defined using either the single criteria of receded near point of convergence (NPC) of $\geq 10 \mathrm{~cm}^{24,30,37,43}$ or the multiple criteria recommended by the convergence insufficiency research study (CIRS) groups ${ }^{38-40}$ (Table 1). The prevalence range of CI for school aged children (aged between 6 and 18 years) when defined using the single criteria is $6-18 \% 0^{24,30,37,43}$ while the range using the multiple criteria is between 2.1 and $33 \%{ }^{26,28,32,40-41}$. The distribution of exophoria $\geq 2$ pd ranged between $1.3 \%$ and $22 \%{ }^{31,37,44}$ and esophoria ranged between $1.4 \%$ and $11.9 \% 0^{31,37,44}$. This review of the literature reveals variations in the reported prevalence estimates which may be related to the differences in measurement techniques and criteria applied to define the parameters studied. Identified limitations with previous studies include lack of recent studies on the epidemiology of NVAs as well as limited studies on how ethnicity, race, age, gender, geographic location, or socio-economic status influence near vision anomalies. Furthermore, no known studies were found that specifically investigated the prevalence of NVAs in high school children in South Africa. Two studies ${ }^{33-34}$ in South Africa studied aspects of accommodative and vergence anomalies in primary school children (PSC). In Metsing and Ferreira, ${ }^{33} 80$ learners aged between 8 and 13 years were enrolled after seven were excluded due to their poor responses to all the tests performed. The concerns of inter-examiner variability were not addressed as data 
was collected by several clinicians under the guidance of the principal investigator. Moodley ${ }^{34}$ retrospectively analyzed data from a vision screening of 264 children aged 6-13 years in Durban. The findings from both studies ${ }^{33-34}$ are integrated in the discussion section of this paper. Although both studies ${ }^{33-34}$ are relevant in providing baseline information on accommodative and vergence functions on school children in South Africa, the convenience sampling method which limits generalization of findings were used to select participants for the studies and both studies ${ }^{33-34}$ were conducted on PSC. Given that accommodative and vergence anomalies are functionally related to near point activities ${ }^{1,3,7}$, PSC are less prone to engage in intense near task demands which may be the trigger to $\mathrm{NVAs}^{45}$. Also, younger children may experience more difficulty responding to the subjectiveness of some tests used to assess accommodative and vergence functions. In addition, aspects of these studies ${ }^{33-34}$ were limited in the classification of accommodative and vergence anomalies which are clinically meaningful when classified as syndromes ${ }^{40,46}$. Thus, the prevalence of aspects of near vision anomalies such as refractive errors in relation to near vision functions, convergence insufficiency $(\mathrm{CI})$, convergence excess $(\mathrm{CE})$ and fusion vergence dysfunction (FVD) as well as accommodative insufficiency (AI), accommodative excess (AE) and infacility (AIF) and the distribution of heterophoria in high school children in South Africa are unknown.

A consideration of the clinical importance, the possible negative consequences of NVAs and a lack of data on the epidemiology of NVAs in high school children in South Africa has motivated the current study. A study on the epidemiology of NVAs in high school children is significant firstly, as it will enable identification of learners in the population that require intervention and secondly guide clinicians and researchers in knowing the expected prevalence, distribution and characteristics of NVAs. Epidemiological studies will provide clues towards determining the possible etiology of anomalies by identifying the associated risk factors. The current study is a pilot project and the aim was to investigate the feasibility of conducting a larger study, and to derive prevalence estimates which will guide determination of appropriate sample size for the main study. The feasibility objectives of the pilot study include a review of the process, resources management, instrumentation, logistics, scientific questions and participant issues ${ }^{47}$.

\section{Methods}

This pilot study was a cross sectional descriptive study designed to provide preliminary empirical information on prevalence, distribution and characteristics of NVAs in a population of high school children in South Africa. The study protocol was approved by the Biomedical Research Ethics Committee of the University of KwaZulu-Natal Durban, South Africa. Written informed consent for access to the schools was obtained from the Department of Education and from the school principals. Information leaflets, the consent and assent forms which were written in both the English language and the learners' native language (isiZulu). They were distributed to all learners and their parents. In the information leaflets, the purpose and procedure of the study were explained. Parental consent was obtained from parents and assents from learners. The purpose and test protocols were also verbally explained to the learners before the eye examinations started. Overall, the conduct of the study complied with the Declaration of Helsinki regarding research on human subjects ${ }^{48}$.

The study was conducted at a high school in a rural location in uMhlathuze municipality in the province of KwaZulu-Natal, South Africa. The uMhlathuze municipality is an administrative area in the uThungulu district of KwaZulu-Natal and the City of uMhlathuze is situated on the north-east coast of the province of KwaZulu-Natal, approximately 170 kilometers north-east of Durban ${ }^{49}$.

\section{Participants}

The target population for the main study is Black high school learners in the uMhlathuze municipality. The participants for the pilot study comprised 65 learners (30 males and 35 females) who were selected using simple random sampling. To be included, learners had to be developmentally normal children, of Black race and of either gender. Learners were excluded from the study if they had any systemic conditions, were on any systemic medication that may affect accommodation or vergence functions, had amblyopia, suppression, strabismus, ocular diseases 
or nystagmus.

\section{Materials and procedure}

The school principal provided a room at the school venue where the visual examinations were conducted. To ensure that the participants understood all the instructions, trial testing was performed for seemingly complex tests such as accommodative facility and fusional vergences. The applicability of all the techniques was confirmed by performing the techniques on patients (of similar age ranges as in present study) seen at the first author's practice over a period of six weeks. This exercise revealed that fusion vergence measurements using the von Graefe technique to be difficult to comprehend by the learners and therefore not feasible to use. Following random selection, learners were given consent forms for their parents to sign. The response rate was 100\% as all learners had parental consent and assented to participate. Due to the nature of the study, it was not possible to mask the researchers from the participants. However, attempts were made to minimize bias. First, the symptoms questionnaires were handed to the learners to complete at the entry point of the data collection. To avoid collaborations and thereby minimize bias, learners were meant to sit aside from one another. The assistant who collected the learners' demographic details worked independent of the optometrists and therefore the optometrist was not aware of the symptoms reported by the learners. In addition, validated optometric equipment was used, test procedures and testing conditions across participants were standardized and where relevant, an average of three test measurements was taken. Data were collected by experienced optometrists who were skilled in measuring all the parameters of interest while the principal investigator ensured that the testing protocol was adhered to. The statisticians who analyzed the data were not optometrists and therefore free of bias towards the data.

All vision tests were performed over a period of two weeks in the month of February 2013, usually from 8 am till 1:30 pm daily except weekends. The compliance to the data collection protocol during the testing was monitored by the principal investigator.

All vision tests were performed in two stations by the first author and another optometrist with one examiner assigned to a station thus eliminating possible inter-examiner variability. The instruments and the procedures used in this study followed the techniques described in standard optometric texts ${ }^{4,50-51}$ and were used in previous studies ${ }^{26-44}$. All near tests were performed at $40 \mathrm{~cm}$ and the tests performed in the first station include visual acuity which was assessed using the Logarithm of Minimum Angle of Resolution (LogMAR) chart (Precision Vision, USA) at both distance and near. Refractive errors were determined objectively using an autorefractor (MRK3100; Huvitz, Gunpo, Gyeonggi, South Korea) and subjectively with a monocular fogging method with cross-cylinder, followed by binocular balancing to a maximum plus for best VA. Subjective data was used for analyses as in other studies ${ }^{29,32,52}$ as the objective findings would be inappropriate for the subsequent near tests. Spherical lenses (2 D) were used to screen for latent hyperopia as cycloplegia was not applied. Cycloplegic refraction was not performed as it would have disrupted the sequence of testing for near vision functions and it was not always easy as school principals were sometimes unwilling to permit learners leaving class frequently (a previous study ${ }^{20}$ in Durban highlighted parental refusal to the use of cycloplegia on their children). Suppression was evaluated at near using the Worth4-dot test (Bernell Corporation, Mishawaka Inc, USA), stereopsis was assessed using the Random dot stereo test (Vision Assessment Corporation). Ocular motility was assessed using the Broad-H-test and recorded as SAFE (smooth, accurate, and with full extent of movement) and rated from 4 to 1 ( 4 for smooth and accurate, 3 represents a fixation loss, 2 for fixation losses and 1 for more than two obvious jerky movements).

All vision tests in station two were measured three times and the average was used for analysis. The unilateral cover test was performed first to rule out strabismus. The following tests were performed with the refractive results in place. Distance and near heterophoria was evaluated using the von Graefe (VG) technique in a specially-designed phoropter tripod. The amplitude of accommodation (AA) was measured monocularly and binocularly using Donder's push-up method with a Royal Air Force (RAF) near point rule 
(Clement Clarke International). The accommodative facility (AF) was assessed monocularly and binocularly using a $\pm 2 \mathrm{D}$ lens flippers ${ }^{4,50-51}$ without suppression control as learners who suppressed were excluded. Accuracy of accommodation was evaluated using the monocular estimation method (MEM) ${ }^{4}, 50-51$ and relative accommodation was measured using the phoropter ${ }^{4}, 50-51$. Ocular health status was evaluated using a direct ophthalmoscope , $^{50-51}$. Fusional vergences at near were measured using a horizontal prism bar. It was difficult to obtain reports on blur points therefore only break and recovery points were used in the analysis. Similar to some published studies ${ }^{28,52-53}$ the von Graefe technique was used to assess heterophoria and prism bars to measure fusional vergences. In contrast to the von Graefe vergence technique, the prism bar is a preferred tool in a screening setting as it is quicker, allows viewing eye movements and is easier for school-aged children ${ }^{54}$.

A 20-point symptoms questionnaire, modified from the Convergence Insufficiency Symptoms Survey (CISS) ${ }^{55}$ and the College Of Optometrist in Vision Development (COVD) ${ }^{56}$ quality of life symptoms survey was administered to the learners at the initial stage of the examination before visual acuity was assessed. The CISS ${ }^{55}$ consists of 15 questions about symptoms and is categorized into two subscales. The performance-related subscale which consisted of six symptoms including loss of concentration, loss of place with reading, reading slowly, trouble remembering what was read, getting sleepy while reading and having to re-read lines. The eye-related subscale consists of nine symptoms specific to visual function or asthenopic-type complaints (such as eyes hurt, diplopia, blurred vision, headaches, eye tiring, sore, words that move and jump, uncomfortable eyes and pulling sensations around the eyes) ${ }^{55}$. Although non-validated symptoms survey were used in some studies ${ }^{31,57-58}$, in the current study, the questionnaire was modified by selecting fewer questions from the COVD survey and expanded on a few questions from the CISS survey. The purpose of the symptoms questionnaire was only to gain insight into the frequency and types of asthenopic symptoms prevalent among high school children, which may impair efficient reading. Hence, the researchers did not deem it necessary to validate the questionnaire as that was not the main focus of the study. Also, neither the CISS survey nor the COVD embraced all the potential symptoms likely to be associated with our parameters of interest and several questions on the COVD questionnaire were not relevant to the purpose of this study. The responses from the survey were analyzed in relation to the clinical measures and gender of the children. The non-validation of the survey may not have compromised the validity of the findings as the symptoms scores were not used to predict or diagnose CI.

\section{Classifications of outcome variables}

The criteria for refractive findings were based on specified cut-offs ${ }^{59-60}$ and the refractive status was assigned based on the eye with the greater refractive error $(\mathrm{RE})^{59-60}$. Spherical equivalent (sphere $+1 / 2$ cylinder) was used only to summarize the REs and were not used for further analysis. As shown in Table 1 various criteria was used to classify accommodative $^{4}, \quad 18,26,28,29-35$, convergence ${ }^{4}, \quad 25-41$ anomalies and heterophoria, ${ }^{25,31}$. For CI, the CIRS ${ }^{38-40}$ criteria were applied while the other criteria were used to classify CE and FVD ${ }^{4,28-29,32}$.

\section{Data analysis}

All data were entered on Microsoft Excel and reviewed by the first author and all analyses were done using the Statistical Package for Social Sciences (SPSS) version 21. Descriptive statistics were presented with means and standard deviations while distributions of variables were presented in text and histograms. Proportions and corresponding 95\% confidence intervals were presented as an estimate of the prevalence. The Chi-squared test was used to investigate possible relations between outcome variables and gender. Kendall's Tau correlation, a nonparametric test which makes no assumptions about the distribution of data was used to investigate possible associations between variables and symptoms. The data for stereopsis, Worth-four-dot and Broad-H-test were not reported in detail as they were used only for exclusion purposes. 
Table 1: Diagnostic criteria for refractive, accommodative and vergence anomalies

\section{Variable /Diagnostic criteria}

\section{Refractive Errors}

Hyperopia $\geq 0.75 \mathrm{D}$

Myopia $\geq-0.50 \mathrm{D}$

Astigmatism $\geq-0.75 \mathrm{D}$ cylinder

Anisometropia $\geq 0.75$ sphere or cylinder between both eyes

Emmetropia $<-0.50 \mathrm{D},<0.75 \mathrm{D}$ and $<-0.75 \mathrm{D}$ cylinder

\section{Accommodative Anomalies}

\section{Accommodative Insufficiency}

(1) Reduced amplitude of accommodation. Push-up monocular accommodative amplitude at least 2 D below

Hofstetter's calculation for minimum amplitude: 15 - 0.25 Age (years)

(2) High monocular estimation method (MEM) retinosocopy $>0.75$

(3) Fails monocular accommodative facility (MAF) testing with $-2 \mathrm{D}<6 \mathrm{cpm}$.

(4) Fails binocular accommodative facility (BAF) testing with $-2 \mathrm{D},<3 \mathrm{cpm}$

\section{Accommodative Excess}

(1) Low MEM $<0.25$

(2) Difficulty clearing $2 \mathrm{D}$ with MAF, $<6 \mathrm{cpm}$.

(3) Fails BAF with $2 \mathrm{D}<3 \mathrm{cpm}$.

Accommodative Infacility

(1) Fails BAF and MAF using \pm 2 D lenses, monocular $<11 \mathrm{cpm}$, binocular $<8 \mathrm{cpm}$

(2) Positive relative accommodation $(\mathrm{PRA})<-2 \mathrm{D}$, Negative relative accommodation $(\mathrm{NRA})<+2.37 \mathrm{D}$.

\section{Convergence anomalies}

\section{Convergence Insufficiency}

(1) Exophoria at near

(2) Exophoria @ near greater than at far $[(\geq 4$ prism diopter $(\mathrm{pd})]$

(3) Insufficient positive fusional vergence (PFV) (i) fails Sheard's criteria or (ii) poor positive fusional vergence $(\mathrm{PFV})$ at near $\leq 12 \mathrm{pd}$. Base out $(\mathrm{BO})$ to blur or $\leq 15 \mathrm{pd}$ BO break/

(4) receded NPC $\geq 7.5 \mathrm{~cm}$ break or $\geq 10.5 \mathrm{~cm}$ recovery.

\section{Convergence insufficiency diagnostic groups:}

(1) Low suspect CI (exophoria@ near which is $\geq 4$ pd greater in magnitude than the distance phoria and clinical sign \#1).

(2) High suspect CI (exophoria at near, and two signs or clinical sign 1 and 2 plus 3 or 4 (that is, low suspect plus clinical sign 3 or 4$)$.

(3) Definite CI (all clinical signs must be present).

\section{Convergence excess}

(1) Significant esophoria at near $\geq 2 \mathrm{pd}$.

(2) Reduced negative fusional vergence (NFV) at near $<8 / 16 / 7$ for blur/break/recovery (1 of 3 )

(3) High MEM ( $\geq 0.75 \mathrm{D})$

\section{Fusional vergence dysfunction}

(1) Reduced PFV and NFV

(2) Normal phoria

(3) Low refractive errors

Heterophoria (near) Exo: $\geq 6$ pd, Eso: $\geq 4$ pd 


\section{Results}

A total of 65 learners (30 males and 35 females) were initially enrolled for the study. One learner had amblyopia and was excluded; one was aged 22 years while the third suppressed the right eye and was excluded. Eventually only 62 learners participated in the study. The ages of the participants ranged between 13 and 19 years with mean age and standard deviation of $17 \pm 1.43$ years. Of the 62 participants, $33(54 \%)$ were female (mean age and $\mathrm{SD}=17.3 \pm 1.2$ years) and $29(46 \%)$ were male (mean age and $\mathrm{SD}=17.2 \pm$ 1.6 years). There was no significant difference in age between male and female cohorts $(p=0.78)$.

About $95 \%$ of participants had unaided visual acuity of 0 LogMAR visual acuity (6/6) and better for both distance and near. Only the right eye refractive values were analyzed as mean spherical equivalent right and the left eyes were the same $(-0.25 \pm 0.46)$ range $(-0.75-0.75)$. The mean and standard deviation for the outcome variables were: NPC $5.30 \pm 1.35 \mathrm{~cm}$ (range, 5-14 cm), monocular AA, $17.96 \pm 3.23 \mathrm{D}$ (range 3-20 D), MAF $10.7 \pm 2.22 \mathrm{cpm}$ (range 5-15 cpm), AR 0.13 D (range 0.25-0.75 D), PFC $33.91 \pm$ $12.87 \mathrm{pd}$ (range 4-45 pd), exophoria, $3.07 \pm 2.03 \mathrm{pd}$ (range $0-12 \mathrm{pd}$ ), esophoria, $2.67 \pm 1.5 \mathrm{pd}$ (range $0-6$ $\mathrm{pd})$.

Analysis of the association between gender and the clinical signs revealed no significant relationship between gender and the following clinical signs: NPC, $\chi^{2}=1.76, p=0.19$, exophoria at near, $\chi^{2}=0.71$, $p=0.87, \mathrm{PFC}, \chi^{2}=1.52, p=0.47, \chi^{2}=0.37 p=0.54$ monocular AA, $\chi^{2}=0.79 p=0.37$, binocular AA, $\chi^{2}$ $=1.41 p=0.234$, binocular AF with $2 \mathrm{D}$ lenses, $\chi^{2}$ $=0.168 p=0.68$, binocular AF using $2 \mathrm{D}$ lenses $\chi^{2}=$ $0.39 p=0.532$. Further analysis was performed to determine whether the sometimes or always response was more frequent among the participants in various symptoms types. The sometimes response was higher in all symptoms categories. More females consistently responded to the always response in all 20 symptoms questions (Figure 1) whereas males responded higher to the sometimes response in 11 out of 20 questions while females responded to 9 out of 20 symptoms questionnaire (Figure 2).

Table 2: Prevalence of outcome variables

\begin{tabular}{lll}
\hline Classification & $\boldsymbol{n}$ & $\mathbf{\%}$ Frequency \\
\hline Refractive anomalies & & \\
Myopia (RE) & 3 & 4.83 \\
Hyperopia (RE) & 1 & 1.61 \\
Astigmatism (RE) & 1 & 1.61 \\
Anisometropia & 0 & 0 \\
Accommodative anomalies & & \\
Accommodative Insufficiency & 1 & 1.61 \\
Accommodative excess & 0 & 0 \\
Accommodative infacility & 1 & 1.61 \\
Poor accommodative facility (monocular) & 15 & 25 \\
Poor accommodative facility (binocular) & 4 & 6.7 \\
$\quad$ Vergence anomalies & & \\
Receded NPC & 2 & 3.2 \\
Convergence Insufficiency & & \\
Low suspect & 10 & 16 \\
High suspect & 0 & 0 \\
Definite & 1 & 1.61 \\
Convergence excess & & \\
Fusional vergence dysfunction & 2 & 3.22 \\
Distribution of heterophoria & 0 & 0 \\
Exophoria & & \\
Esophoria & 3 & 5.76 \\
\hline
\end{tabular}



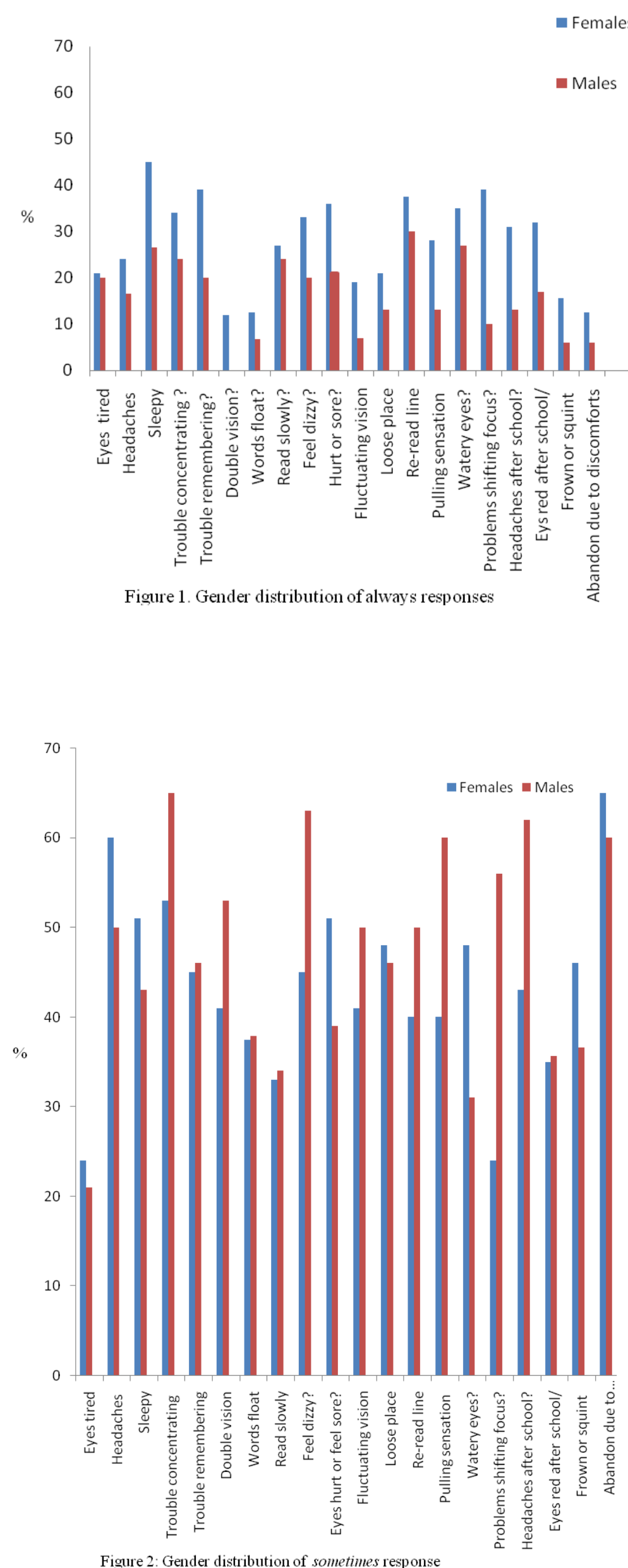

The possible relationships between the clinical measures and the 20 points symptoms were investigated using Kendall Tau correlation tests. The analysis revealed an inverse correlation between the following clinical signs and symptoms for the following questions; q13 (Do you have to re-read the same line of words when you read?) in relation to binocular AF with positive lenses $(r=-0.368, p$ $<0.05) \mathrm{q} 13$ and binocular AF with negative lenses $(r$ $=-0.298, p<0.05)$, q15 (Do your eyes water when you read?) and binocular AF with negative lenses $(r=$ $-0.247, p<0.05)$. For the remaining clinical measures and symptoms, the responses were similar. Overall, there was no relationship between clinical signs and symptoms as the never responses were statistically greater in the anomalous subgroup than the normal subgroup in most cases.

\section{Discussion}

In this pilot study, the feasibility of conducting a large scale study on near vision anomalies in high school children was investigated. A higher prevalence of convergence anomalies than accommodative and refractive anomalies was found. This pilot project also revealed that it is possible to conduct the main study on a large scale with minor modifications. In relation to other studies, the $1.6 \%$ prevalence of hyperopia is comparable to findings from other studies which ranged from 1.24 to $1.81 \%^{20-21,23}$, the $4.8 \%$ prevalence of myopia is similar to the $4.05 \%$ reported by Niroula and Saha 22 but lower than the $2.9 \%$ reported by Naidoo et al $^{20}$. The $1.6 \%$ prevalence of astigmatism is similar to $1.14 \%$ reported by Niroula and Saha ${ }^{22}$ but lower than $6.7 \%$ reported by Naidoo et $a l^{20}$. In relation to near vision performance, refractive corrections alleviate asthenopic symptoms common in binocular anomalies ${ }^{25,30}$. Similar to RE results, the findings on accommodative anomalies were relatively low. The $1.61 \%$ prevalence of $\mathrm{AI}$ is lower than that from other studies ${ }^{26,28,32}$ and the differences in the prevalence estimates may be due to differences in measurement technique and criteria used in various studies. The finding on AIF $(1.6 \%)$ is lower than the $13.4 \%$ reported by Shin et al ${ }^{32}$ even though Shin et al ${ }^{32}$ applied multiple criteria to define AIF as in current study. Shin et $a l^{32}$ studied primary school children. Metsing and Ferreira ${ }^{33}$ reported that $12.3 \%$ of the 
participants had poor AF whereas Moodley ${ }^{34}$ found a much higher proportion of poor binocular AF (30\%) which is comparable to our findings of $25 \%$ for poor $\mathrm{AF}$, although they ${ }^{32-34}$ studied younger children aged between 6 and 13 years. No participant had AE in the current study. Overall, the prevalence estimates on the syndromes of accommodative anomalies (AI, AIF and AE) in the current study were lower than findings from studies on non-African populations ${ }^{26,28,32}$. The small sample size may limit firm conclusion from the current study and differences in findings across studies may be related to differences in measurement techniques and criteria applied in the studies.

For convergence anomalies, if the remote NPC criterion is applied to define $\mathrm{CI}$, the $3.2 \%$ in the current study is lower than the reported range of between 6 and $18 \%$ from previous studies ${ }^{30-31,36-37,43}$. The difference here may be related to methodological differences involving the ages of participants ${ }^{61}$. Defining CI using the NPC single criteria may limit interpretation as usually $\mathrm{CI}$ is defined as a syndrome of clinical signs $^{40}$. For studies that applied the CIRS criteria, the findings of the current study on CI [low suspect $\mathrm{n}=10(16 \%)$; high suspect (none); definite $1(1.6 \%)]$ are comparable to a pilot study of 35 school children by Rouse $e t a l^{38}$ in some ways: a higher prevalence of low suspect CI, $\mathrm{n}=8(33.3 \%)$, high suspect, $\mathrm{n}=1$ $(4.2 \%)$, definite $n=1(4.2 \%)$ but contrasts with the findings from other studies: Rouse et $a l^{40}$ reported the total prevalence of CI to be $21.4 \%$; classified as: low suspect (8.4\%), high suspect (8.8\%) and definite (4.2\%). Marran et $a l^{28}$ reported the prevalence of CI was $14.7 \%$ for CI types 1 and 2. In the study by Borsting et a ${ }^{26}$ the total prevalence of CI of $17.3 \%$ (4.6\% had three signs of $\mathrm{CI}$ and $12.7 \%$ had two signs of $\mathrm{CI}$ ). For convergence excess, the prevalence of $3.2 \%$ found in the current study is higher than the $0.8 \%$ reported by Borsting et al $^{27}$ but lower than the $2.4 \%$ reported by Shin et al ${ }^{32}$ and $5 \%$ by Marran et $a l^{28}$. For heterophoria at near target distances, only the study by Abdi et l $^{31}$ applied a similar definition as for the current study ( $\geq 6 \mathrm{pd}$ for exophoria and $\geq 4$ pd for esophoria) although they measured heterophoria using the cover test technique. The reported prevalence of near exophoria was $8.8 \%$ and esophoria was $1.4 \%$. The findings of the current study of $5.7 \%$ exophoria are lower than those reported by Abdi et $a l^{31}$ whereas the $1.6 \%$ prevalence of esophoria is comparable to theirs. The differences in techniques and criteria between the two studies limit comparisons.

In summary, given that the nature and amount of near tasks affect near vision performance ${ }^{4,57,62-63} \mathrm{a}$ high prevalence of low suspect (borderline cases) $\mathrm{CI}$ and relatively high frequency of near horizontal heterophoria suggests that such participants might develop more definite CI when their near task demands increase. For the symptoms, these findings of never responses being statistically greater in the anomalous subgroup than the normal subgroup suggest that the asthenopic symptoms are not good indicators of NVAs. A reason for a lack of correlation between symptoms and clinical measures (a finding consistent with other reports ${ }^{57,63}$ ), may be that the symptoms of asthenopia are nonspecific and not differentiated on the basis of the causative factor ${ }^{62-63}$. Other possible reasons are that some subjects may avoid near activities to eliminate symptoms ${ }^{63}$ or that the learners do not engage in intensive near point activities to generate symptoms.

For the always response, females responded more positively in all 20 symptoms questions and almost equal number of positive sometimes response with males (Figure 1). This suggests that females may have more symptoms more frequently than males or that more females are more likely to report their symptoms more frequently than males. Overall, the relatively low prevalence of near vision anomalies may mean that the learners did not engage in much near point activities which may trigger anomalies or that it may be necessary to measure the accommodative and vergence parameters after near work. On feasibility objectives, the pilot study revealed that all techniques were age-appropriate as learners understood the test instructions and questionnaires adequately.

There are some limitations which may preclude firm conclusions to be drawn from the study findings. The relatively small sample size limits generalization. The symptoms questionnaire was not validated. In addition, the use of the von Graefe technique to assess heterophoria and fusional vergence would have allowed for consistency in approach although the findings were used only to categorize the threesign CI which had only one case (1.6\%) frequency. However, this study was a screening survey requiring fast and simple techniques and therefore the use of a more complex technique such as the von Graefe fusional reserves technique in a non-clinic setting would be complicated for the learners to understand 
and perform. Despite these possible limitations, the study is relevant as a feasibility project to researchers who wish to design similar studies and findings from the study provide preliminary data on the prevalence of NVAs in high school learners. The study design will be modified according to the concerns as stated above. For example, the validated version of the CISS questionnaire is already in use to collect data related to convergence insufficiency in the main study.

\section{Conclusion}

Although the prevalence of NVAs was relatively low, convergence anomalies were found to be higher than refractive and accommodative anomalies. Symptoms of asthenopia were prevalent in this sample of school children although was not strongly associated with near vision anomalies. This pilot project reveals that it is highly feasible to conduct the study on a large scale with some modifications. Identification and referrals for near vision anomalies are important steps towards diagnosis and treatment which will minimize discomfort with reading and subsequent poor performance which may be associated such anomalies.

\section{References}

1. Garzia R. [chapter title?] In: Scheiman, M. Rouse, M. Optometric management of learning-related vision problems. 1st edition. Missouri: Mosby, 2006.

2. Bodack M, Chun I, Krumholtz, I. An analysis of vision screening data from New York City public schools. Optometry 201081 476-484.

3. Cacho- Martinez P, Garcia-Munoz A, Ruiz-Cantero MT. Do we really know the prevalence of accommodative and non-strabismic binocular dysfunctions? J Optom 20103 185-197.

4. Scheiman M, Wick B. Clinical management of binocular vision: Heterophoric, accommodative and eye movement disorders, 3rd edition. Philadelphia: JB Lippincott, 2008.

5. Wajuihian SO, Naidoo KS. Dyslexia: An overview. $S$ Afr Optom 201170 89-98.

6. Ritty JM, Solan HA, Cool SJ. Visual and sensory-motor functioning in the classroom: a preliminary report of ergonomic demands. J Am Optom Assoc 199364 238-244.

7. Jimenez R, Gonzalez MD, Perez MA, Garcia JA. Evolution of accommodative functions and developments of ocular movements in children. Ophthal Physiol Opt $20032397-$ 17.

8. Granet D, Gomi C, Ventura R, Miller-Scholte A. The relationship between convergence Insufficiency and
ADHD. Strabismus 200513 163-168.

9. Borsting E, Rouse M, Chu R. Measuring ADHD behaviours in children with symptomatic accommodative dysfunction or convergence insufficiency: a preliminary study. Optometry 200576 588-592.

10. Gronlund MA, Aring E, Landren M, Hellstro A. Visual function and ocular features in children and adolescents with attention deficit hyperactivity disorder, with and without treatment with stimulants. Eye 200721 494-502.

11. Damari DA, Liu J, Smith KB. Visual disorders misdiagnosed as ADHD. Case studies and literature. J Behav Optom 2000 11 87-91.

12. Dusek W, Piersionek BK, McClelland JF. A survey of visual functions in an Austrian population of school-age children with reading and writing difficulties. BMC Ophthal 201010 1-10.

13. Zaba JN. Social, emotional and educational consequences of undetected children's vision problems. J Behav Optom 200112 66-70.

14. Johnson RA, Nottingham D, Stratton R, Zaba J. The vision screening of academically and behaviorally at- risk pupils. J Behav Optom 199671 39-42.

15. Grisham JD, Simons HD. Refractive error and the reading process: a literature analysis. J Am Optom Assoc 198657 44-55.

16. Palomo-Alvarez C. Binocular functions in school children with reading difficulties. Graefe Arch Clin Exp Ophthalmol 2010248 885-892.

17. Wajuihian SO, Naidoo KS. Visual factors and dyslexia: A research review. S Afr Optom 201069 58-68.

18. Scheiman M, Gallaway M, Coulter R, Reinstein F, Ciner E. Prevalence of vision and ocular disease conditions in a clinical pediatric population. J Am Optom Assoc 199667 193- 202.

19. Bennett GR, Blondin M, Ruskiewics J. Incidence and prevalence of selected visual conditions. J Am Optom Assoc 198253 647- 656.

20. Naidoo KS, Raghunandan A, Mashige P, Govender P, Holden BA, Gopal P, Pokharel GP, Ellwein LB. Refractive errors and visual impairments in African children in South Africa. Invest Ophthal Vis Sci 200344 3764-3770.

21. Ovenseri-Ogbomo GO, Omuemu V. Prevalence of refractive error among school children in the Cape Cost Municipality, Ghana. 2010 Clin Optom 2 59-66.

22. Niroula DR, Saha CG. Study on the refractive errors of school going children of Pokhara city in Nepal. Kath Univ Med J 2009 7 67-72.

23. Quek TPL, Chua CG, Chong CS. Prevalence of refractive errors in teenage high school students in Singapore. Ophthal Physiol Opt 200424 47-55.

24. Harris P. Learning-related visuals problems in Baltimore city: A long-term program. J Optom Vis Dev 200233 75115.

25. Dwyer $\mathrm{P}$, Wick B. The influence correction upon disorders of vergence and accommodation. Optom Vis Sci 199572 224-232.

26. Borsting, E. Rouse, M. Deland, P. Hovett, S. Kimura, D. Park, M. Association of symptoms and convergence and 
accommodative insufficiency in school-age children. Optometry 200374 25-34.

27. Borsting E, Rouse M, De Land P. The prevalence of convergence excess in school-aged children Optom Vis Sci 200380169 (Meeting abstract).

28. Marran LF, De Land PN, Nguyen AL. Accommodative insufficiency is the primary source of symptoms in children diagnosed with convergence insufficiency. Optom Vis Sci 2006 E281-E289.

29. Lara F, Cacho P, Garcia A, Megias R. General binocular disorders: prevalence in a clinic population. Ophthal Physiol Opt 200121 70-74.

30. Abdi S, Rydberg A. Asthenopia in schoolchildren, orthoptic and ophthalmological findings and treatment. Doc Ophthalomol 2005111 65-72.

31. AbdiS, Lennerstrand G, Pansell T, Rydberg A. Orthoptic findings and asthenopia in a population of Swedish schoolchildren aged 6 to 16 years. Strabismus 200816 4755.

32. Shin HS, Park SC, Park CM. Relationship between accommodative and vergence dysfunction and academic achievement for primary school children. Ophthal Physiol Opt 200929 615-624.

33. Metsing IT, Ferreira JT. Accommodation and vergence status among the 3rd and 4th graders in mainstream school in Gauteng. S Afr Optom 201271 22-31.

34. Moodley VR. Amplitude, facility and accuracy of accommodation in primary school population. S Afr Optom 200867 147-154.

35. Wick B, Hall P. Relation among accommodative facility, lag, and amplitude in elementary school children. Am J Optom Physiol Opt 198764 593-598.

36. Walters J. Portsea modified clinical technique: Results from an expanded optometric screening protocol for children. Aust J Optom 198467 176-218.

37. Jungham B, Keily P, Crewther DP, Crewther SG. Referral rates for a functional vision screening among a large cosmopolitan sample of Australian children. Ophthal Physiol Opt 200222 10-25.

38. Rouse MW, Borsting E, Hyman L, Hussein M. Pilot study to evaluate convergence insufficiency in a school-aged population: Poster \# 54 ( Bv-323). Optom Vis Sci 199572 218.

39. Rouse, M. Hyman, L. Hussein, M. Solan, H. Frequency of convergence insufficiency in optometry clinic settings. Convergence insufficiency and reading study (CIRS) Group. Optom Vis Sci 199875 88-96.

40. Rouse MW, Borsting E, Hyman L, Hussein M, Solan H. Frequency of convergence insufficiency in optometry clinic settings. Optom Vis Sci 199975 88-96.

41. Letourneau J, Ducic S. Prevalence of convergence insufficiency among elementary school children. Can J Optom 198850 194-197.

42. Robinson B, Acorn CJ, Millar CC, Lyle WM. The prevalence of selected ocular diseases and conditions. Optom Vis Sci 199774 79-91.

43. Letourneau J, Lapierre N, Lamont A. The relationship between convergence insufficiency and school achievement.
Am J Optom Physiol Opt 197956 18-22.

44. Aring E, Gronlund MA, Andersson S. Strabismus and binocular functions in a sample of Swedish children aged 4-15 years. Strabismus 200513 55-61.

45. Cooper J, Duckman R. Convergence insufficiency: incidence, diagnosis, and treatment. J Am Optom Assoc 197849 673-80.

46. Daum KM. Accommodative dysfunction. Doc Ophthalmol 198355 177-198

47. Thabane L, Ma J, Chu R, Cheng J, Ismalia A, Rios LP, Robson R, Thabane M, Giangregorio L, Goldsmith CH. A tutorial on pilot studies: the what, why and how $B M C \mathrm{Med}$ Res Meth 201010 2-10.

48. Declaration of Helsinki. http:/www.who.int/bulletin/ archives/79\%284\%29373.

49. uMhlathuze municiaplity Http://www.richemp.org.za/ TulipuMhlathuzeInternet/

50. Eskridge JB, Amos JF, Bartlett JD. Clinical procedures in Optometry. New York: JP Lippincott Company, 1991.

51. Carlson NB, Kurtz D. Clinical Procedures in Ocular Examination $3^{\text {rd }}$ ed. New York: McGraw Hill Professional Publishers, 2004.

52. Porcar E. Martinez-Palomera, A. Prevalence of general binocular dysfunctions in a population of university students. Optom Vis Sci 199774 111-113.

53. Nisted I, Maagaard ML, Bek T. Independence between age-related changes in refraction, accommodation and convergence in primary school children. Scand J Optom Vis Sc 20136 6-9.

54. Scheiman M. Rouse M. Optometric management of learning-related vision problems 2nd edition. Missouri: Mosby, 2006.

55. Borsting EJ, Rouse MW, Mitchell GL, Scheiman M, Cotter SA, Cooper J. Validity and reliability of the revised Convergence Insufficiency Symptom Survey in children aged 9 to 18 years. Optom Vis Sci 200380 832-838.

56. Maples WC. Frequency and types of pediatric symptoms in a clinical population. J Optom Vis Dev 2010412 74-80.

57. Cohen Y, Segal O, Barkana YM, Lederman R, Zadok D, Pras E, Morad Y. Correlation between asthenopic symptoms and different measurements of convergence and reading comprehension and saccadic fixation eye movements. Optometry 201081 28-34.

58. Sterner, B. Gellerstedt, M. Sjöström, A. (2006). Accommodation and the relationship to subjective symptoms with near work for young school children. Ophthal Physiol Opt 26 148-155.

59. Hashemi H, Fotouhi A, Mohammad K. The age- and gender-specific prevalences of refractive errors in Tehran Eye study. Ophthal Epid 200411 213-225.

60. Ip JM, Robaei D, Kifley A, Wang JJ, Rose KA, Mitchel P. Prevalence of hyperopia and association with eye findings in 6 and 12-years olds. Ophthalmology 2008115 678-685.

61. Rouse MW. Management of binocular anomalies: Efficiency of vision therapy in the treatment of accommodative deficiencies. Am J Optom Physiol Opt 198764 415-420.

62. Sheedy JE, Hayes J, Engle J. Is all asthenopia the same? Optom Vis Sci 200380 732-739. 
63. Bade A, Boas M, Gallaway M, Mitchell L, Scheiman M, Kulp MT. Relationship between clinical signs and symptoms of convergence insufficiency. Optom Vis Sci 201390 988-995. 